\title{
A Type of Arithmetic Labels about Circulating Ring
}

\author{
Ergen Liu, Dan Wu, and Kewen Cai
}

School of Basic Sciences, East China Jiaotong University, Nanchang, P.R. China, 330013

leg_eg@sina.com

\begin{abstract}
The graph composed with several rings is a kind of important and interesting graph, many scholars studied on the gracefulness of this kind of the graph, The reference [1] is given the gracefulness of $m$ kinds $C_{4}$ with one common point. In this paper, we researched the arithmetic labels of four kinds graph: $C_{8,1, n}, C_{8,2, n}, C_{8,3, n}, C_{8,4, n}$, and we proved they are all $(d, 2 d)$--arithmetic graph.
\end{abstract}

Keywords: Arithmetic graph, labeling of graph, $C_{8, i, n}$.

\section{Introduction}

The graph in this paper discussed are undirected, no multiple edges and simple graph, the unorganized state of definitions and terminology and the symbols in this graph referred to reference[2][3].

There are two kinds of labels of the graph: one is the reduced label, is to say that in order to get the label of one edge you should reduce the endpoints of the edge; the other is additive label, for the same you should acttive the endpoints of one edge to get the label of the edge. For example, the well-known of "Gracefulness" is reduced. the "Compatible labels" is additive. In 1990, B.D.Achaya and S.M.Hegde import the concept of "Arithmetic lables"(referred to reference[2]), which is a more extensive additive label, it have applied value on solution to question of the joint ventures in rights and obligations.

Definition 1.1. For $G=(V, E)$, if there is a mapping $f$ ( called the vertices $v$ of the label ) from $V(G)$ to the set of nonnegative integer $N_{0}$, meet:

(1) $f(u) \neq f(v)$, which $u \neq v$, and $u, v \in V(G)$;

(2) $\{f(u)+f(v) \mid u v \in E(G)\}=\{k, k+d, \cdots, k+(q-1) d\}$.

Then we call graph $G$ is a $(k, d)$--arithmetic graph. 


\section{Main Results and Certification}

Theorem 2.1. $C_{8,1, n}$ is a $(d, 2 d)$--arithmetic graph.

Proof. As the graph shown on fig.1.

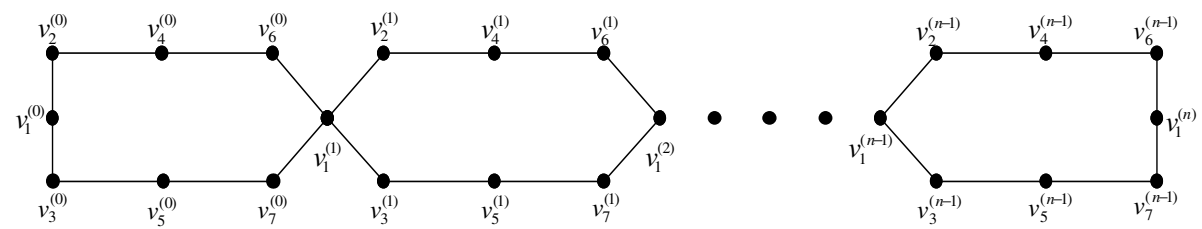

Fig. 1. The Graf $C_{8,1, n}$

Label all vertices as follows:

$$
\begin{array}{ll}
f\left(v_{1}^{(i)}\right)=8 i d(i=0,1,2, \cdots, n), & f\left(v_{2}^{(i)}\right)=8 i d+d(i=0,1,2, \cdots, n-1), \\
f\left(v_{3}^{(i)}\right)=8 i d+3 d(i=0,1,2, \cdots, n-1), & f\left(v_{4}^{(i)}\right)=8 i d+6 d(i=0,1,2, \cdots, n-1), \\
f\left(v_{5}^{(i)}\right)=8 i d+2 d(i=0,1,2, \cdots, n-1), & f\left(v_{6}^{(i)}\right)=8 i d+5 d(i=0,1,2, \cdots, n-1), \\
f\left(v_{7}^{(i)}\right)=8 i d+7 d(i=0,1,2, \cdots, n-1) . &
\end{array}
$$

Now we proof that the mapping $f$ is arithmetic labeling of $C_{8,1, n}$.

We can see the mapping $f$ meet $f(u) \neq f(v)$ which $u \neq v$ and $u, v \in V\left(C_{8,1, n}\right)$.

Next we prove $\left\{f(u)+f(v) \mid u v \in E\left(C_{8,1, n}\right)\right\}$ is an arithmetic progression in the way of mathematical induction.

When $n=1$

Then $f\left(v_{1}^{(0)}\right)=0, f\left(v_{2}^{(0)}\right)=d, f\left(v_{3}^{(0)}\right)=3 d, f\left(v_{4}^{(0)}\right)=6 d, f\left(v_{5}^{(0)}\right)=2 d$, $f\left(v_{6}^{(0)}\right)=5 d, f\left(v_{7}^{(0)}\right)=7 d, f\left(v_{1}^{(1)}\right)=8 d$.

Therefore $\left\{f(u)+f(v) \mid u v \in E\left(C_{8,1,1}\right)\right\}$

$$
\begin{aligned}
= & \left\{f\left(v_{1}^{(0)}\right)+f\left(v_{2}^{(0)}\right), f\left(v_{1}^{(0)}\right)+f\left(v_{3}^{(0)}\right), f\left(v_{3}^{(0)}\right)+f\left(v_{5}^{(0)}\right),\right. \\
f\left(v_{2}^{(0)}\right)+ & f\left(v_{4}^{(0)}\right), f\left(v_{5}^{(0)}\right)+f\left(v_{7}^{(0)}\right), f\left(v_{4}^{(0)}\right)+f\left(v_{6}^{(0)}\right), f\left(v_{6}^{(0)}\right)+f\left(v_{1}^{(1)}\right), \\
f\left(v_{7}^{(0)}\right)+ & \left.f\left(v_{1}^{(1)}\right)\right\} \\
= & \{d, 3 d, 5 d, 7 d, 9 d, 11 d, 13 d, 15 d\}
\end{aligned}
$$

is an arithmetic progression, and the common difference is $2 d$. 
Suppose when $n=k-1$, we know

$\left\{f(u)+f(v) \mid u v \in E\left(C_{8,1, k-1}\right)\right\}=\{d, d+1 \times 2 d, d+2 \times 2 d, \cdots, d+(8 k-9) \times 2 d\}$

is an arithmetic progression, and the common difference is $2 d$.

Then when $n=k$

$\left\{f(u)+f(v) \mid u v \in E\left(C_{8,1, k}\right)\right\}$

$=\left\{f(u)+f(v) \mid u v \in E\left(C_{8,1, k-1}\right)\right\} \bigcup\left\{f\left(v_{1}^{(k-1)}\right)+f\left(v_{2}^{(k-1)}\right), f\left(v_{1}^{(k-1)}\right)+f\left(v_{3}^{(k-1)}\right)\right.$, $f\left(v_{3}^{(k-1)}\right)+f\left(v_{5}^{(k-1)}\right), f\left(v_{2}^{(k-1)}\right)+f\left(v_{4}^{(k-1)}\right), f\left(v_{5}^{(k-1)}\right)+f\left(v_{7}^{(k-1)}\right)$,

$\left.f\left(v_{4}^{(k-1)}\right)+f\left(v_{6}^{(k-1)}\right), f\left(v_{6}^{(k-1)}\right)+f\left(v_{1}^{(k)}\right), f\left(v_{7}^{(k-1)}\right)+f\left(v_{1}^{(k)}\right)\right\}$

$=\{d, d+1 \times 2 d, d+2 \times 2 d, \cdots, d+(8 k-9) \times 2 d\} \bigcup\{d+(8 k-8) \times 2 d$,

$d+(8 k-7) \times 2 d, d+(8 k-6) \times 2 d, d+(8 k-5) \times 2 d, d+(8 k-4) \times 2 d$,

$d+(8 k-3) \times 2 d, d+(8 k-2) \times 2 d, d+(8 k-1) \times 2 d\}$

$=\{d, d+1 \times 2 d, d+2 \times 2 d, \cdots, d+(8 k-1) \times 2 d\}$

is an arithmetic progression, and the common difference is $2 d$.

In sum for the arbitrary $n \in N_{0}$, the mapping $f: V\left(C_{8,1, n}\right) \rightarrow N_{0}$ meet:

(1) $f(u) \neq f(v)$ when $u \neq v$ and $u, v \in V\left(C_{8,1, n}\right)$;

(2) $\left\{f(u)+f(v) \mid u v \in E\left(C_{8,1, n}\right)\right\}=\{d, d+1 \times 2 d, d+2 \times 2 d, \cdots, d+(8 n-1) \times 2 d\}$.

Therefore $C_{8,1, n}$ is a $(d, 2 d)$--arithmetic graph.

Theorem 2.2. $C_{8,2, n}$ is a $(d, 2 d)$--arithmetic graph.

Proof. As the graph shown on fig.2.
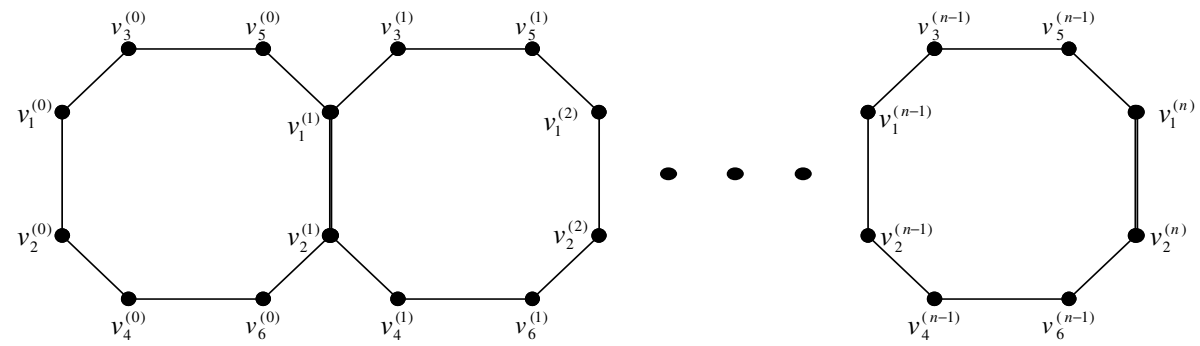

Fig. 2. The Graf $C_{8,2, n}$ 
Label all vertices as follows:

$$
\begin{array}{ll}
f\left(v_{1}^{(i)}\right)=7 i d(i=0,1,2, \cdots, n), & f\left(v_{4}^{(i)}\right)=7 i d+6 d(i=0,1,2, \cdots, n-1), \\
f\left(v_{2}^{(i)}\right)=7 i d+d(i=0,1,2, \cdots, n), & f\left(v_{5}^{(i)}\right)=7 i d+2 d(i=0,1,2, \cdots, n-1), \\
f\left(v_{3}^{(i)}\right)=7 i d+3 d(i=0,1,2, \cdots, n-1), & f\left(v_{6}^{(i)}\right)=7 i d+5 d(i=0,1,2, \cdots, n-1) .
\end{array}
$$

Now we proof that the mapping $f$ is arithmetic labeling of $C_{8,2, n}$

We can see the mapping $f$ meet $f(u) \neq f(v)$ which $u \neq v$ and $u, v \in V\left(C_{8,2, n}\right)$.

Next we prove $\left\{f(u)+f(v) \mid u v \in E\left(C_{8,2, n}\right)\right\}$ is an arithmetic progression in the way of mathematical induction.

When $n=1$

Then $f\left(v_{1}^{(0)}\right)=0, f\left(v_{2}^{(0)}\right)=d, f\left(v_{3}^{(0)}\right)=3 d, f\left(v_{4}^{(0)}\right)=6 d, f\left(v_{5}^{(0)}\right)=2 d$, $f\left(v_{6}^{(0)}\right)=5 d, f\left(v_{1}^{(1)}\right)=7 d, f\left(v_{2}^{(1)}\right)=8 d$.

Therefore $\left\{f(u)+f(v) \mid u v \in E\left(C_{8,2,1}\right)\right\}$

$$
=\left\{f\left(v_{1}^{(0)}\right)+f\left(v_{2}^{(0)}\right), f\left(v_{1}^{(0)}\right)+f\left(v_{3}^{(0)}\right), f\left(v_{3}^{(0)}\right)+f\left(v_{5}^{(0)}\right),\right.
$$

$$
\begin{aligned}
& f\left(v_{2}^{(0)}\right)+f\left(v_{4}^{(0)}\right), f\left(v_{5}^{(0)}\right)+f\left(v_{1}^{(1)}\right), f\left(v_{4}^{(0)}\right)+f\left(v_{6}^{(0)}\right), f\left(v_{6}^{(0)}\right)+f\left(v_{2}^{(1)}\right), \\
& \left.f\left(v_{1}^{(1)}\right)+f\left(v_{2}^{(1)}\right)\right\} \\
& \quad=\{d, 3 d, 5 d, 7 d, 9 d, 11 d, 13 d, 15 d\}
\end{aligned}
$$

is an arithmetic progression, and the common difference is $2 d$.

Suppose when $n=k-1$, we know

$$
\left\{f(u)+f(v) \mid u v \in E\left(C_{8,2, k-1}\right)\right\}=\{d, d+1 \times 2 d, d+2 \times 2 d, \cdots, d+(7 k-7) \times 2 d\}
$$

is an arithmetic progression, and the common difference is $2 d$.

Then when $n=k$

$$
\begin{gathered}
\left\{f(u)+f(v) \mid u v \in E\left(C_{8,2, k}\right)\right\} \\
=\left\{f(u)+f(v) \mid u v \in E\left(C_{8,2, k-1}\right)\right\} \bigcup\left\{f\left(v_{1}^{(k-1)}\right)+f\left(v_{3}^{(k-1)}\right),\right. \\
f\left(v_{3}^{(k-1)}\right)+f\left(v_{5}^{(k-1)}\right), f\left(v_{2}^{(k-1)}\right)+f\left(v_{4}^{(k-1)}\right), f\left(v_{5}^{(k-1)}\right)+f\left(v_{1}^{(k)}\right), \\
\left.f\left(v_{4}^{(k-1)}\right)+f\left(v_{6}^{(k-1)}\right), f\left(v_{6}^{(k-1)}\right)+f\left(v_{2}^{(k)}\right), f\left(v_{1}^{(k)}\right)+f\left(v_{2}^{(k)}\right)\right\}
\end{gathered}
$$




$$
\begin{aligned}
& \quad=\{d, d+1 \times 2 d, d+2 \times 2 d, \cdots d+(7 k-7) \times 2 d\} \cup\{d+(7 k-6) \times 2 d, \\
& d+(7 k-5) \times 2 d, d+(7 k-4) \times 2 d, d+(7 k-3) \times 2 d, d+(7 k-2) \times 2 d, \\
& d+(7 k-1) \times 2 d, d+7 k \times 2 d\} \\
& \quad=\{d, d+1 \times 2 d, d+2 \times 2 d, \cdots, d+7 k \times 2 d\}
\end{aligned}
$$

is an arithmetic progression, and the common difference is $2 d$.

In sum for the arbitrary $n \in N_{0}$, the mapping $f: V\left(C_{8,2, n}\right) \rightarrow N_{0}$ meet:

(1) $f(u) \neq f(v)$ when $u \neq v$ and $u, v \in V\left(C_{8,2, n}\right)$;

(2) $\left\{f(u)+f(v) \mid u v \in E\left(C_{8,2, n}\right)\right\}=\{d, d+1 \times 2 d, d+2 \times 2 d, \cdots, d+7 n \times 2 d\}$.

Therefore $C_{8,2, n}$ is a $(d, 2 d)$--arithmetic graph.

Theorem 2.3. $C_{8,3, n}$ is a $(d, 2 d)$--arithmetic graph .

Proof. As the graph shown on fig.3.

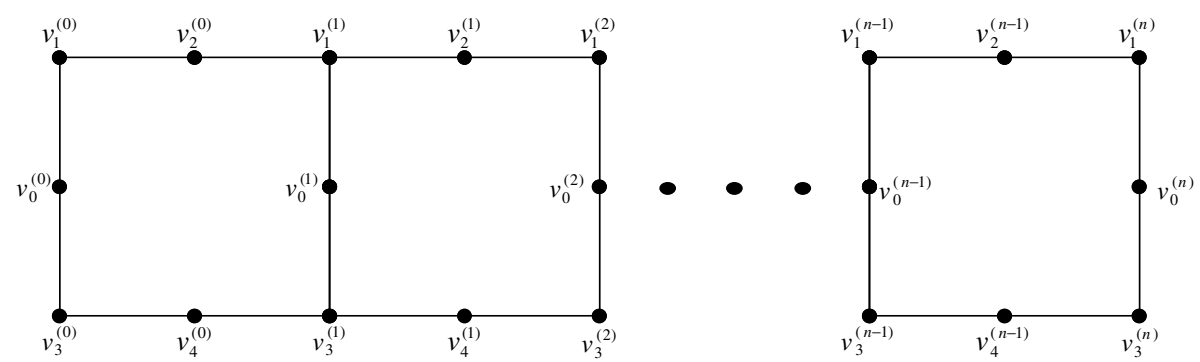

Fig. 3. The Graf $C_{8,3, n}$

Label all vertices as follows:

$$
\begin{array}{ll}
f\left(v_{0}^{(i)}\right)=8 i d(i=0,1,2, \cdots, n), & f\left(v_{1}^{(i)}\right)=4 i d+d(i=0,1,2, \cdots, n), \\
f\left(v_{3}^{(i)}\right)=4 i d+3 d(i=0,1,2, \cdots, n), & f\left(v_{2}^{(i)}\right)=8 i d+6 d(i=0,1,2, \cdots, n-1), \\
f\left(v_{4}^{(i)}\right)=8 i d+2 d(i=0,1,2, \cdots, n-1) . &
\end{array}
$$

Proof in imitation of Theorem 2.1.

For the arbitrary $n \in N_{0}$, the mapping $f: V\left(C_{8,3, n}\right) \rightarrow N_{0}$ meet:

(1) $f(u) \neq f(v)$ when $u \neq v$ and $u, v \in V\left(C_{8,3, n}\right)$;

(2) $\left\{f(u)+f(v) \mid u v \in E\left(C_{8,3, n}\right)\right\}=\{d, d+1 \times 2 d, d+2 \times 2 d, \cdots, d+(6 n+1) \times 2 d\}$. 
By (1), (2) we know the mapping $f$ is arithmetic labeling of $C_{8,3, n}$. Therefore $C_{8,3, n}$ is a $(d, 2 d)$--arithmetic graph.

Theorem 2.4. $C_{8,4, n}$ is a $(d, 2 d)$--arithmetic graph.

Proof. As the graph shown on fig.4.
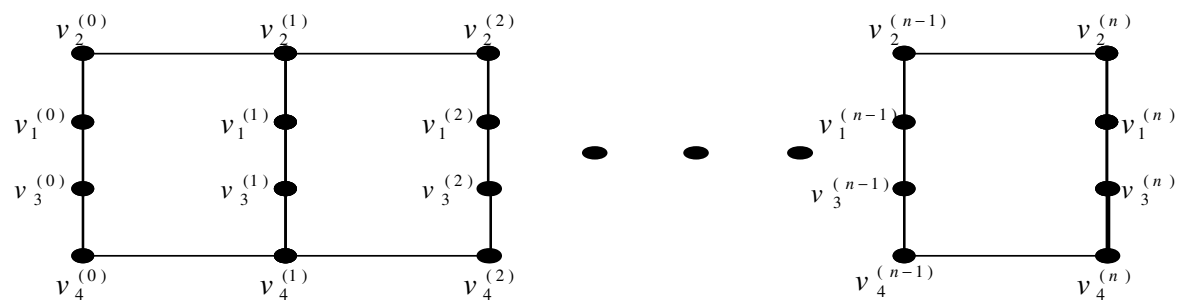

Fig. 4. The Graf $C_{8,4, n}$

Label all vertices as follows:

$$
\begin{array}{ll}
f\left(v_{1}^{(i)}\right)=5 i d(i=0,1,2, \cdots, n), & f\left(v_{2}^{(i)}\right)=5 i d+d(i=0,1,2, \cdots, n), \\
f\left(v_{3}^{(i)}\right)=5 i d+3 d(i=0,1,2, \cdots, n), & f\left(v_{4}^{(i)}\right)=5 i d+2 d(i=0,1,2, \cdots, n) .
\end{array}
$$

Proof in imitation of Theorem 2.2.

For the arbitrary $n \in N_{0}$, the mapping $f: V\left(C_{8,4, n}\right) \rightarrow N_{0}$ meet:

(1) $f(u) \neq f(v)$ when $u \neq v$ and $u, v \in V\left(C_{8,4, n}\right)$;

(2) $\left\{f(u)+f(v) \mid u v \in E\left(C_{8,4, n}\right)\right\}==\{d, d+1 \times 2 d, d+2 \times 2 d, \cdots, d+(5 n+2) \times 2 d\}$.

By (1), (2) we know the mapping $f$ is arithmetic labeling of $C_{8,4, n}$. Therefore $C_{8,4, n}$ is a $(d, 2 d)$--arithmetic graph.

\section{Labels of Some Special Graph}

In order to explan the correctness of the aforementioned labels, we give the arithmetic labeling of $C_{8,1,3} \quad C_{8,2,3} \quad C_{8,3,3}$ and $C_{8,4,3}$. 
(1) The arithmetic labeling of $C_{8,1,3}$ on fig.5.

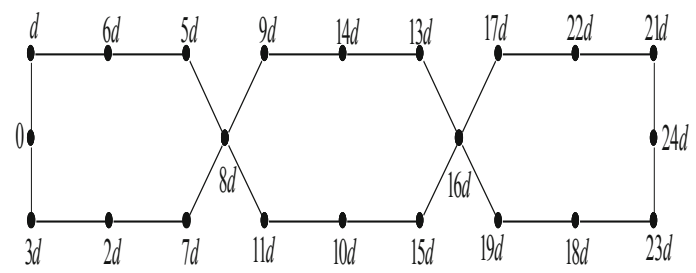

Fig. 5. The Graf $C_{8,1,3}$

(2) The arithmetic labeling of $C_{8,2,3}$ on fig.6.

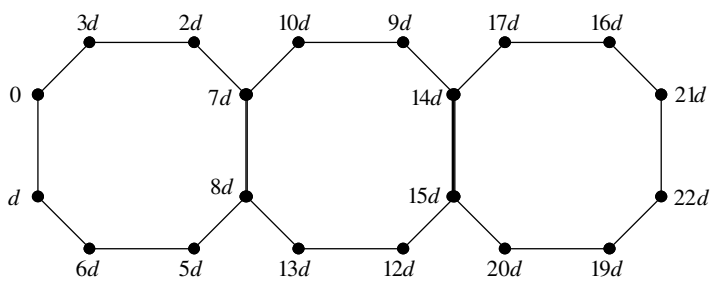

Fig. 6. The Graf $C_{8,2,3}$

(3) The arithmetic labeling of $C_{8,3,3}$ on fig.7.

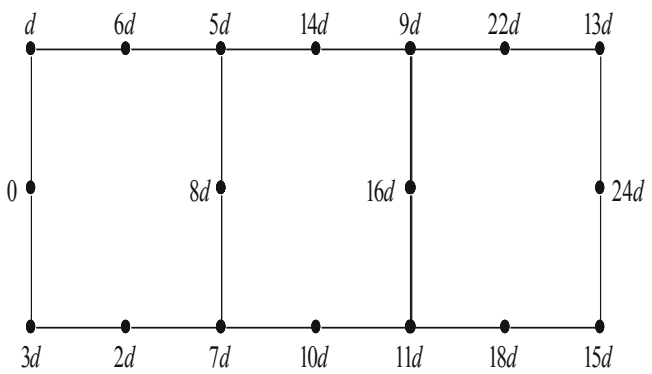

Fig. 7. The Graf $C_{8,3,3}$ 
(4) The arithmetic labeling of $C_{8,4,3}$ on fig.8.

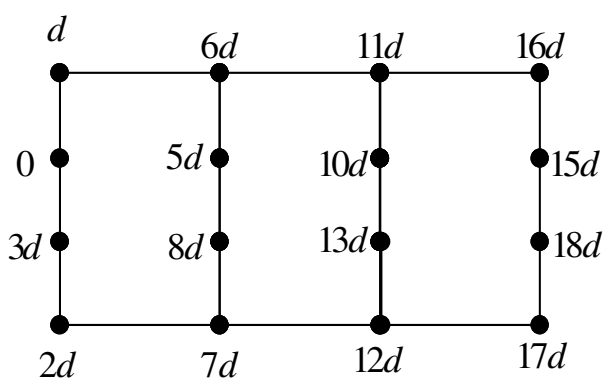

Fig. 8. The Graf $C_{8,4,3}$

\section{References}

[1] Ma, K.: Gracefulnss. The press of technology, Beijing (1991)

[2] Chartrand, G., Lesniak, L.: Graphs and Digraphs. Wadsworth and Brooks/Cole, Monterey (1996)

[3] Bondy, J.A., Murty, U.S.R.: Graph Theory with Applications. Elsevier North-Holland (1976)

[4] Liu, E.-g., Wu, D., Cai, K.-w.: Two Graphs Arithmetic Labeling. Journal of East China Jiaotong University 26(5), 89-92 (2009)

[5] Achaya, B.D., Hegde, S.M.: Arithmetic graphs. Journal of Graph Theory 18(3), 275-299 (1990) 